\title{
EREBEA
}

Revista de Humanidades

y Ciencias Sociales

Núm. 7 (2017), pp. 109-125

ISSN: 0214-0691

\section{TEMPORA TURBULENTA. LA LUTTE CONTRE LES DISSIDENCES URBAINES À TRAVERS L'EXEMPLE DES RELATIONS AD LIMINA DE MAYENCE AU DÉBUT DU XVII ${ }^{\mathrm{E}}$ SIÈCLE}

Étienne Bourdeu

Université François Rabelais, Tours (Francia)

RESUMEN

Las relaciones ad limina permiten ver cómo la contrarreforma entra en aplicación en los territorios católicos. A partir del análisis de estos documentos producidos para el arzobispado de Maguncia, este artículo pretende estudiar los intentos para reconstruir la autoridad política y religiosa de los arzobispos de Maguncia pero también cómo ellos intentan borrar las huellas de las disidencias en sus territorios. Este proceso no se puede disociar de un importante esfuerzo de construcción y de un reforzamiento de los lazos con otros príncipes católicos. Así, los intentos para acabar con las disidencias permiten que los arzobispos de Maguncia tengan una importancia que supera los problemas de política interna y de la religión. También se encarnan en un discurso de exaltación del poder arzobispal.

Palabras Clave

Maguncia, Reforma católica, relación ad limina, siglo 17.

Fecha de recepción: 13 de mayo de 2017 Fecha de aceptación: 10 de nov. de 2017

\section{ABSTRACT}

As historical sources, the relations ad limina make clear how the Counter-Reformation came into effect within catholic territories; they also show the means used by the archbishops to erase the remnants of past dissidences. This study intends to analyse the attempts to build and reinforce the political and religious authority of the archbishops of Mainz. Such attempts cannot be dissociated from an important enterprise of architectural building and from the creation of close relations with other catholic princes. They can also manifest themselves as a speech intending to exalt the power of the archbishops. Thus, to put an end to dissidences allows the archbishops of Mainz to be part of processes that go beyond questions of internal or religious politics.
KeYwords

Mainz, Catholic reform, relation ad limina, $17^{\text {th }}$ century. 

En 1604, apprenant la mort l'archevêque de Mayence Johann Adam von Bicken, l'ambassadeur hispanique dans le Saint-Empire, Guillén de San Clemente, dresse un rapide portrait du défunt prélat au consejo de Estado : "cétait un grand serviteur de la maison d'Autriche; cela a été une grande perte" ${ }^{1}$. Bien qu'élu à peine trois ans plus tôt à la tête de la principauté électorale de Mayence, Johann Adam von Bicken semble bien avoir été un personnage apprécié des agents de la Monarchie Catholique.

L'explication de ce jugement positif ne se trouve pas exclusivement dans les prérogatives institutionnelles - connues des Hispaniques - faisant des archevêques de Mayence "le directeur de toutes les choses de l'Empire et le chancelier de celui-ci ; celui qui, en période de sede vacante, réunit tous les électeurs et qui est le premier qui doive y consentir" ${ }^{2}$. Il faut y voir aussi le résultat d'une activité constante des princes de Mayence pour affirmer leur autorité face aux autres électeurs impériaux, en nouant notamment des liens privilégiés avec la papauté et avec des princes étrangers à l'espace germanique ${ }^{3}$. Pourtant, dans cette volonté d'affirmer une autorité dépassant le simple cadre d'une principauté territoriale et s'inscrivant dans un complexe politique plus vaste et de nature impériale, un élément interne a peu été pris en compte par l'historiographie : la lutte contre les dissidences.

Il est vrai que s'intéresser à ces dernières revient à s'interroger sur une ambiguïté liée à la définition même du terme de "dissidence". En effet, le mot s'applique aussi bien à une divergence doctrinale, politique comme confessionnelle, qu'à un groupe d'individus séparés du reste d'une communauté par l'effet de cette même divergence doctrinale ; en somme, la notion de dissidence s'applique aussi bien

1 Archivo General de Simancas [AGS], Estado [E], legajo [leg.] 708, lettre de Guillén de San Clemente au consejo de Estado, 26 janvier 1604 : "Era gran serv[id]or de la casa de Austria, ha sido gran pérdida".

2 Idem : "El directorio de todas las cosas del Imp[eri]o y cançiller del y el que en sede vacante junta todos los electores para la electión y es el primero que [h] a de consentir en ella".

3 C. Roll, "Sin lieb sy auch eyn kurfurst.... Zur Rolle Bertholds von Henneberg in der Reichsreform”, dans P. C. Hartmann (éd.), Kurmainz, das Reichserzkanzleramt und das Reich am Ende des Mittelalters und im 16. und 17. Jahrhundert. Stuttgart: Franz Steiner Verlag, 1998, p. 5-43 ; E. Schubert, "Der Mainzer Kurfürst als Erzkanzler im Spätmittelalter", dans P. C. Hartmann (éd.), Der Mainzer Kurfürst als Reichserzkanzler. Funktionen, Aktivitäten, Ansprüche und Bedeutung des zweitens Mannes im Alten Reich. Stuttgart: Franz Steiner Verlag, 1997, pp. 77-97; G. May, "Der Erzbischof von Mainz als Primas", dans P. C. Hartmann (éd.), Der Mainzer Kurfürst als Reichserzkanzler..., pp. 35-76. 
à la cause (le désaccord) qu’à ses conséquences (la division). Malgré cette relative indécision, l'usage de cette notion a été porteur d'un réel renouveau épistémologique en histoire, particulièrement en ce qui concerne la question des affrontements confessionnels. Elle a ainsi permis d'analyser de façon plus fine et plus dynamique la constitution, le fonctionnement et l'évolution de groupes confessionnels ${ }^{4}$. À cette occasion, la dissidence apparaît être à l'opposé de l'idée d'un phénomène sectaire et de repli sur soi ; cette inscription dans la sphère publique est même une des conditions d'accès à la légitimité, pour celui qui s'affirme dans la dissidence comme pour celui qui la combat ${ }^{5}$. De ce point de vue, la dimension spatiale de l'étude de la dissidence est cruciale pour rendre compte des mécanismes de son fonctionnement et il faut relever le fait que le thème des dissidences a été essentiellement étudié dans un cadre urbain ; il est donc également révélateur du renouveau d'un champ historiographique qui s'intéresse à la question de l'identité (notamment sociale et culturelle) de l'espace urbain, constitutive de la nature même du lien politique au sein des communautés urbaines ${ }^{6}$.

Pour ce qui est de la principauté électorale de Mayence, il est possible d'étudier les dissidences apparues dans les principales villes de ce territoire à partir d'une source originale, les relations ad limina. Il s'agit de visites effectuées par un évêque à celui de Rome pour informer ce dernier de l'état temporel et spirituel d'un diocèse. Nées avec la réforme grégorienne du XI ${ }^{\mathrm{e}}$ siècle, les relations ad limina Apostolorum sanctorum Petri et Pauli sont remises en vigueur avec la constitution pontificale Pontifex romanus de 1585. Deux ans plus tard, avec la constitution Immensa aeterni Dei, Sixte Quint confie à la congrégation du concile, chargée de l'interprétation et de l'application des canons du concile de Trente, le soin de veiller à ce que ces visites ou, à leur place, des rapports soient effectivement adressés au siège romain. En raison de l'éloignement de certains diocèses, ceux-ci sont rapidement répartis en groupes en fonction de la périodicité à laquelle ils doivent envoyer leurs rapports à Rome. Cet échelonnement prévoit ainsi une régularité de trois ans pour les évêchés les plus proches de Rome (Italie et îles adjacentes) jusqu'à dix ans pour les diocèses asiatiques et américains ${ }^{7}$. L'espace germanique

4 S. Gal, Grenoble au temps de la Ligue. Étude politique, sociale et religieuse d'une cité en crise (vers 1562 - vers 1598). Grenoble: Presses Universitaires de Grenoble, 2000 ; M. Monge, Des communautés mouvantes. Les sociétés des frères chrétiens en Rhénanie du Nord, Juliers, Berg, Cologne vers 1530-1694. Genève: Droz, 2015.

5 H. Hermant, Guerres de plumes : publicité et cultures politiques dans l'Espagne du XVII siècle. Madrid: Casa de Velázquez, 2012.

6 É. Crouzet-Pavan et É. Lecuppre-Desjardin, "Les mots de l'identité. La ville, le pouvoir et l'écrit en Europe aux derniers siècles du Moyen Âge", dans Histoire urbaine, vol.3, nº 35 (2012), pp. 5-12.

$7 \mathrm{M}^{a} \mathrm{M}$. Cárcel Ortí, Relaciones sobre el estado de las diócesis valencianas. Valence: Generalitat Valenciana, Conselleria de Cultura, Educació i Ciència, 1989; E. W. Zeeden, "Visitationsforschung und Kirchengeschichtsschreibung", dans Theologische Revue, 87 (1991), pp. 353-366. 
doit observer une régularité de quatre ans entre chaque rapport. À partir de 1588, on peut considérer, pour reprendre les mots de Philippe Boutry et de Bernard Vincent, que "la visite ad limina est née dans sa forme moderne".

Si les visites et rapports ad limina de Mayence s'inscrivent, grosso modo, dans ce cadre, elles marquent également les spécificités de l'Église impériale. La première visite date de 1609 , soit vingt-quatre ans après l'institution de cette pratique. De la même façon, le rythme quadriennal n'est pas respecté puisque les quatre premiers rapports s'échelonnent en 1609, 1615, 1621 et 1629 . On peut ici raisonnablement penser que "le vacarme des guerres et des armes, ainsi que les dangers des chemins d'Italie", pour reprendre la formule utilisée en 1629 par l'archevêque Anselm Casimir Wambold von Umstadt, ont rendu périlleux et peu souhaitables d'entreprendre un voyage à travers des régions touchées par la guerre de Trente ans ${ }^{9}$. D'ailleurs, de manière significative, aucun rapport n'est envoyé entre 1629 et 1658, c'est-à-dire au moment du regain d'intensité du conflit dans l'espace impérial et des deux occupations de Mayence par les troupes suédoises en 1630 et en 1644 .

Pour ce qui est de leur contenu et de leur forme, chacune de ces visites est constituée des lettres de créances des commissaires envoyés par l'archevêque de Mayence, d'une lettre adressée au pape ou au cardinal présidant la congrégation du concile qui explique les raisons pour lesquelles l'archevêque n'a pu se déplacer et qui rappelle sa soumission au pontife romain et, enfin, du rapport du diocèse proprement dit. Entre 1609 et 1629, ce dernier n'a pas encore de forme fixe. Si les deux visites qui inaugurent les règnes de Johann Schweikard von Cronberg et Anselm Casimir Wambold von Umstadt, en 1609 et 1629, sont les plus détaillées, celles de 1615 et 1621 sont plus succinctes et se contentent d'insister sur les nouveautés notables qui concernent le diocèse : "sera passé en revue ce qui touche à l'accroissement du culte divin qui a eu lieu pendant ce temps" 10 .

Peu utilisés dans une telle perspective, ces documents constituent néanmoins un observatoire intéressant des dissidences dans les espaces urbains de la principauté de Mayence. En effet, les archevêques y évoquent aussi bien les différends politiques et confessionnels auxquels ils peuvent être confrontés que leurs tentatives pour y remédier. Le premier cas de figure est fréquemment euphémisé, notamment pour éviter de donner à voir une trop grande fragilité. Le second, et

8 P. Boutry et B. Vincent (éds.), Les Chemins de Rome. Les visites ad limina à l'époque moderne dans l'Europe méridionale et le monde hispano-américain (XVI - XIX' siècle). Rome: École Française de Rome, 2002, p. 2.

9 Archivio Segreto Vaticano [ASV], Congregazione del concilio, Relationes Dioecesum [Relat. Dioec.] 479, sans titre, 7 novembre 1629, f 164 : "Inter hoc bellorum et armorum strepitus, et itinerum pericula Italicor [um]".

10 ASV, Congregazione del concilio, Relat. Dioec. 479, "Moguntin[a] relatio quadrienny exhibita", 5 juillet 1615, f 55 : "Recensibuntur quae interea ad augmentum dinini cultus". 
sa dimension édilitaire en premier lieu, est en revanche plus longuement développé afin de mettre en scène la capacité des archevêques de Mayence à recréer un consensus dont ils sont les principaux bénéficiaires, tant à l'échelle de leur principauté qu’à celle, plus vaste, du Saint-Empire.

Le travail qui suit se propose donc de réfléchir à la façon dont la lutte contre les dissidences urbaines et sa mise en scène permet aux archevêques de Mayence de construire et de renforcer leur pouvoir : dans quelle mesure cette lutte et cette mise en scène permettent-elles la construction d'un pouvoir territorial qui peut trouver à s'inscrire dans un contexte impérial ?

1. Les espaces urbains de la principauté électorale de Mayence sont à l'image des territoires sur lesquels règne l'archevêque : éclatés et divers. En ce sens, ils constituent un ensemble non seulement composite mais également polycentrique, résultat de l'intégration d'espaces distincts à des moments différents, intégration qui détermine en partie les conditions de l'exercice du pouvoir du prince et sa façon de gérer les dissidences qui ont pu y apparaître ${ }^{11}$. Par facilité, il est possible de distinguer cinq villes qui ont une importance particulière dans la principauté de Mayence : Duderstadt et Heiligenstadt dans l'Eichsfeld, Aschaffenburg, Erfurt et, enfin, Mayence elle-même (voire carte 1).

Illustrant l'idéal-type wéberien de la ville, ces trois dernières constituent des espaces de concentration de richesses et ont une importance économique à l'échelle régionale $^{12}$. En effet, Mayence dispose d'une situation géographique remarquable : elle est située au cœur de ce que Fernand Braudel nomme l'"isthme allemand"13. Cet axe de communication méridien met en contact deux zones opulentes et fortement urbanisées de l'Europe de la première modernité, l'Italie du Nord et les Pays-Bas. Mayence est également établie à l'entrée du massif schisteux rhénan qui concentre et canalise les flux de circulation du Rhin. De ce fait, une source importante de richesses des archevêques de Mayence est la perception des droits de douanes qui ponctuent le cours du fleuve ${ }^{14}$. En ce qui concerne Aschaffenburg, la proximité de Francfort sur le Main, à une cinquantaine de kilomètres, lui permet de tirer profit de l'activité générée par les foires marchandes, puis à partir de 1585 par les foires de change qui s’y établissent. Erfurt correspond également à une zone de relative aisance économique. Rattachée au bassin de l'Elbe grâce à la Gera, cette cité du sud de la Thuringe est

11 P. Cardim et al., Polycentric monarchies: How Did Early Modern Spain and Portugal Achieve and Maintain a Global Hegemony ?. Brighton: Sussex Academic Press, 2012.

12 M. Weber, La Ville. Paris: La Découverte, 2014 [1 ère éd. : 1921].

13 F. Braudel, La Méditerranée et le monde méditerranéen à l'époque de Philippe II. Paris: Armand Colin, 1990, pp. 185-197.

14 Schubert, “Der Mainzer Kurfürst als Erzkanzler...”, p. 79. 


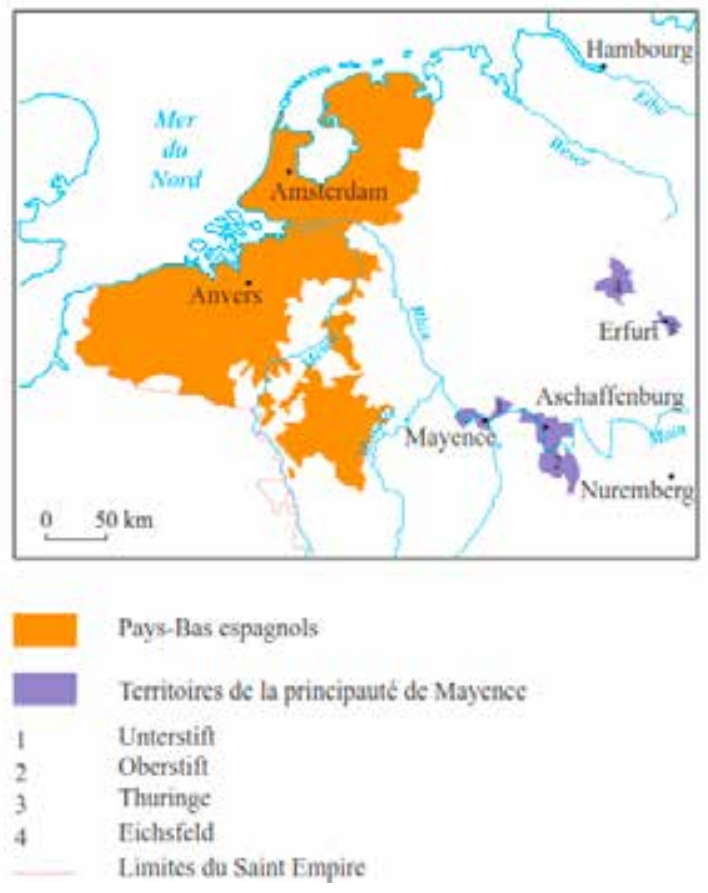

Carte 1 : La principauté ecclésiastique de Mayence et les Pays-Bas espagnols au milieu du $\mathrm{XVI}^{\mathrm{e}}$ siècle (Source : É. Bourdeu, Les archevêques de Mayence et la présence espagnole dans le Saint-Empire, XVI -XVII siècle. Madrid, Casa de Velázquez, 2015, p. 17)

connectée à Hambourg et, d'une manière plus générale, à l'espace hanséatique. C'est aussi une étape de la route reliant Nuremberg, pôle économique de premier ordre dans l'espace germanique du début du XVI ${ }^{\mathrm{e}}$ siècle, à la Baltique et à la mer du Nord ${ }^{15}$. Cette partie de la Thuringe est également un des grands centres européens de production de pastel et une petite industrie textile et métallurgique (notamment grâce au cuivre extrait des mines saxonnes de Mansfeld) s'y développe au commencement du XVI ${ }^{\mathrm{e}}$ siècle ${ }^{16}$. Néanmoins, sa prospérité est surtout due aux foires qui y ont lieu et dont le privilège a été renouvelé par l'empereur Frédéric III en 1477. Mais cette importance commerciale décroît avec la création de la foire de Leipzig et la concession à cette dernière d'un droit d'étape très étendu ${ }^{17}$. Pour

15 H. Kellenbenz, El Desarrollo económico de la Europa continental (1500-1750). Madrid: Siglo XXI, 1977, pp. 143-144.

16 Ibid., p. 95 ; B. Vogler, Le Monde germanique et helvétique à l'époque des réformes. $1517-$ 1618. Paris: SEDES, 1980, p. 218.

17 H. Kellenbenz (éd.), Europäische Wirtschafts- und Sozialgeschichte vom ausgehenden Mittelal- 
autant, cette création n'élimine pas totalement la vieille route commerciale qui va de Hambourg à Nuremberg en passant par Erfurt qui demeure un noud routier important dans l'espace germanique oriental ${ }^{18}$. Ainsi, la principauté électorale de Mayence abrite plusieurs villes qui, si elles ne jouissent pas d'un rayonnement de premier ordre dans l'espace germanique, contribuent néanmoins à polariser l'espace régional où elles se trouvent. De ce point de vue, l'Eichsfeld semble moins remarquable que Mayence ou Erfurt. Située plus au nord que cette dernière et également en Thuringe, c'est une région formée de plateaux et structurée par deux villes de moyenne importance, Heiligenstadt et Duderstadt. Essentiellement rural, l'Eichsfeld est administré par un gouverneur nommé par l'archevêque de Mayence et résidant à Heiligenstadt.

De nombreuses dissidences affectant les espaces urbains de la principauté de Mayence et perceptibles dans les relations ad limina trouvent leurs origines au $\mathrm{XVI}^{\mathrm{e}}$ siècle voire auparavant, dans la seconde moitié $\mathrm{du} \mathrm{Xv}^{\mathrm{e}}$ siècle. En ce sens, on peut parler d'une longue durée des dissidences. Par ailleurs, il est à noter que ces dissidences sont de natures multiples et touchent aussi bien le secteur politique et religieux que l'économique et le social, se cantonnant rarement à un seul de ces domaines ; pour ces raisons également, on peut les considérer comme des phénomènes globaux, permettant d'embrasser des faits humains dans leur totalité.

Une première forme de dissidence, qui a marqué la ville de Mayence de façon durable, est de nature politique. Depuis 1462 et contrairement à d'autres villes d'une taille sensiblement égale, Mayence n'est plus une ville libre d'Empire. À cette date en effet, le nouvel archevêque Adolphe II de Nassau, élu en 1461, avait dû conquérir par la force la ville qui soutenait l'autre candidat au siège épiscopal, Diether von Isenburg. Au terme d'un siège en règle, Adolphe II avait enlevé à Mayence sa justice, son administration et son droit à lever des impôts ${ }^{19}$. La ville est donc soumise à son prince, au prix néanmoins d'une importante perte de ses libertés. De cette façon, le pouvoir territorial des archevêques est durablement accru. Un tel phénomène s'inscrit dans un mouvement plus général d'accroissement du pouvoir temporel de nombreux autres princes germaniques au moment de l'entrée dans l'époque moderne. Toutefois, cet accroissement des prérogatives des archevêques de Mayence ne peut aller beaucoup plus loin : les princes voisins, qu'il s'agisse des comtes de Nassau, des landgraves de Hesse ou des comtes palatins du Rhin, sont de puissants seigneurs qui restreignent les possibilités d'expansion ou d'unification territoriale.

ter bis zur Mitte des 17. Jahrhunderts. Stuttgart: Klett-Cotta, 1986, p. 870.

18 Braudel, La Méditerranée et le monde méditerranéen..., p. 186.

19 A.P. Brück, "Adolf II", dans Neue Deutsche Biographie. Berlin: Duncker \& Humblot, dans Neue Deutsche Biographie. Berlin: Duncker \& Humblot, 1953, vol. 1, pp. 84-85. 
Une deuxième forme de dissidence peut s'observer à Erfurt et implique des éléments aussi bien économiques et sociaux que religieux. Malgré sa richesse, la prospérité d'Erfurt ne contribue que médiocrement à la richesse de la principauté de Mayence. En effet, la fin du Xve siècle voit la multiplication des tensions entre la ville de Thuringe, d'une part, et Mayence qui revendique sa souveraineté (Oberhoheit) sur la cité et l'électeur de Saxe qui fait également valoir ses prétentions, d'autre part. Avec l'entrée dans le $x^{2} I^{\text {e }}$ siècle, le conflit se durcit : alors que le conseil de la ville prétend imposer de nouveaux impôts, les quartiers et les corporations des métiers les plus modestes élisent en 1509 un comité qui exige de participer au gouvernement de la ville. Pendant que le conseil de ville se rapproche de Frédéric III de Saxe, les éléments les plus radicaux pactisent avec l'archevêque de Mayence Uriel von Gemmingen. Finalement, en 1510, un conseil nouvellement élu refuse toute concession à l'archevêque de Mayence ${ }^{20}$. L'unité de la cité vole ainsi en éclats et révèle le manque de cohésion à la fois de différentes catégories sociales de la ville et des élites urbaines. Ces divergences débouchent sur la sollicitation du seigneur légal, l'archevêque de Mayence, mais aussi sur celle d'un autre prince dont la légitimité tient à sa proximité géographique ${ }^{21}$. Toujours est-il qu'au terme de ces dissidences urbaines et pour un peu plus d'un siècle et demi, Erfurt est quasiment indépendante ; ce n'est qu'en 1664 que Johann Philipp von Schönbōrn parvient à rétablir la souveraineté (Landeshoheit) de Mayence ${ }^{22}$. Par ailleurs, il faut aussi relever qu'Erfurt est également une des villes les plus précocement touchées par le luthéranisme et que l'enracinement de la nouvelle confession est durable. Il y a donc ici l'exemple d'une dissidence sociale et politique qui trouve une expression sur le terrain religieux.

Le cas de l'Eichsfeld quant à lui soulève le problème de l'adhésion à la Réforme dans les territoires de la principauté de Mayence et, donc, la question de la dissidence confessionnelle, mais selon des modalités particulières. Si Albert de Brandebourg, archevêque de Mayence de 1514 à 1545, est en partie responsable de la protestation de Luther contre les indulgences (l'archevêque en avait généralisé la vente afin de rembourser un prêt de 21000 ducats contractés auprès des Fugger pour financer son élection à Mayence ${ }^{23}$ ), ses successeurs s'engagent par

20 G. Vogler, Europas Aufbruch in die Neuzeit. 1500-1650. Stuttgart: Ulmer, 2003, p. 50 et pp. 325-326.

21 M. Coquelin, “ L' 'année terrible' d'Erfurt et sa mémoire. Trouble, division et défaite au fondement d'une nouvelle identité urbaine ?", dans Cahiers du CRHQ, mis en ligne le 08/02/2013, consultable sur http://www.unicaen.fr/ $/ \mathrm{mrsh} / \mathrm{crhq} /$ cahiers/page-article.php?num=332\&ch=9, consulté le 09/08/2017.

22 A. Wendehorst, "Johann Philipp von Schönborn”, dans Neue Deutsche Biographie. Berlin : Duncker \& Humblot, 1974, vol. 10, pp. 497-499.

23 H. Grimm, "Albrecht", dans Neue Deutsche Biographie. Berlin: Duncker \& Humblot, 1953, vol. 1, pp. 166-167; M. Arnold, Martin Luther. Paris : Fayard, 2017, p. 105. 
la suite dans un travail de réforme catholique en profondeur, comme on le verra plus tard. Si cette région est touchée précocement par la Réforme luthérienne, une entreprise de recatholicisation vigoureuse est menée à partir de 1575 par Daniel Brendel von Homburg qui fait de l'Eichsfeld une "frontière de catholicité" 24

Ainsi, qu'il s'agisse de Mayence, d'Erfurt ou des villes de l'Eichsfeld, les villes de la principauté ecclésiastique de Mayence sont travaillées par de multiples formes de dissidences. Ces dernières sont certes nombreuses mais elles ne sont, ni par leur nature ni par leur ampleur, plus exceptionnelles que dans d'autres territoires de l'Europe de la première modernité. Il s'agit bien au contraire d'une des nombreuses formes que prend la vie sociale mais qui, lorsqu'elles mettent aux prises le pouvoir, modifient en profondeur la façon d'exercer celui-ci. Il faut enfin remarquer que l'expression de ces désaccords au sein de la principauté de Mayence permet l'ingérence d'acteurs extérieurs, Diether von Isenburg dans le cas du siège de Mayence en 1462 ou l'électeur de Saxe pour Erfurt. De la sorte se trouve posée la question des conséquences des dissidences dans l'insertion politique de l'État territorial de Mayence à l'intérieur du Saint-Empire et celle des moyens mis en œuvre pour résoudre ces problèmes.

2. À lire les relations ad limina, une part importante de l'activité des archevêques de Mayence semble s'exprimer par un travail de construction et de reconstruction. Comme nombre de ces chantiers sont à mettre en relation avec les dissidences passées qui ont pu les opposer aux villes de leur État, il importe de parvenir à établir les bonnes relations entre le travail édilitaire évoqué dans les relations et les divergences à l'œuvre à l'intérieur de la principauté électorale.

En premier lieu, tenus depuis le concile de Trente au devoir de résidence, les évêques catholiques sont obligés d'apporter la preuve qu'ils demeurent parmi leurs ouailles, sauf cas de force majeure ou dispense en théorie exceptionnelle. Les archevêques de Mayence ne dérogent pas à cette ligne et les relations ad limina abordent ouvertement cette question. L'originalité de leur situation tient sans doute au fait que leur lieu de résidence est double. Comme l'indique la relation de 1609, "les sièges de sa résidence ordinaire sont au nombre de deux : l'une, pour l'été, se trouve à Mayence, l'autre, pour l'hiver, se situe à Aschaffenburg"25. Au premier abord, si le choix d'un lieu de résidence dédoublé semble distendre les liens entre l'archevêque et la ville de Mayence, il a néanmoins des raisons

24 C. Duhamelle, "Frontière, territoire, confession : l'exemple de l'Eichsfeld", dans Christine Lebeau (éd.), L'Espace du Saint Empire du Moyen Âge à l'époque moderne. Strasbourg: Presses Universitaires de Strasbourg, 2004, pp. 175-192 ; A.P. Brück, "Daniel Brendel von Homburg", dans Neue Deutsche Biographie. Berlin: Duncker \& Humblot, 1957, vol. 3, p. 507.

25 ASV, Congregazione del concilio, Relat. Dioec. 479, "Relatio oratorum Moguntinorum”, 10 octobre 1609, $\mathrm{f}^{\circ}$ 68v : "Ordinariae residentiae huius sedis loci duo sunt : alter aestiuus Moguntiae, hybernus alter Aschaffenburgi”. 
profondes : d'une part, la ville d'Aschaffenburg est sans doute moins excentrée par rapport au reste du diocèse et surtout de la principauté que Mayence même ; d'autre part, cela signe sans doute le choix des archevêques de se mettre en sécurité vis-à-vis d'une cité qui continue d'être perçue comme remuante et donc menaçante. Le souvenir d'Adolphe II de Nassau et du siège qu'il avait dû mener n'est pas si lointain. Le choix d'un lieu de séjour extérieur à la métropole rhénane peut ainsi en partie s'expliquer par le désir d'échapper à un univers urbain agité.

Pour cette raison, Aschaffenburg est l'objet d'un soin particulier de la part des archevêques de Mayence. À la fois parce que la ville est l'une des deux résidences archiépiscopales et parce qu'elle a subi les dégâts des expéditions militaires du margrave de Brandebourg-Kulmbach Albert Alcibiade au milieu du XVI ${ }^{\mathrm{e}}$ siècle, elle est le théâtre d'une intense activité constructrice. Ainsi, en 1609 Johann Schweikard von Cronberg mentionne le fait

qu' "après le brigandage brandebourgeois qui l'a [la résidence d'Aschaffenburg] laissée détruite et comme rasée [...], il a relancé et a fait entreprendre à partir de ces mêmes décombres et au prix de grandes dépenses des travaux dignes de mémoire, qui ne sont pourtant pas encore arrivés à leur terme, tout cela pour l'honneur et l'usage de l'Église"26.

Les travaux semblent être achevés lors de la relation suivante de 1615 et le même archevêque peut alors se flatter en affirmant : “j”ai construit [...] une nouvelle résidence archiépiscopale dans la ville d'Aschaffenburg à la place de celle détruite par le marquis de Brandebourg" ${ }^{27}$.

Il est intéressant de noter que ces travaux dans la capitale de l'Oberstift n'éloignent nullement les archevêques de la ville de Mayence et cette dernière constitue un des chantiers architecturaux les plus importants du diocèse à en croire les relations ad limina. À ce titre, ils ont soin d'apparaître comme des bâtisseurs ou des restaurateurs. En effet, dès la relation de 1609, Schweikard von Cronberg insiste particulièrement sur les restaurations qu'il a "généreusement" fait entreprendre à Mayence même et qui concernent "des écoles, des églises, des

26 ASV, Congregazione del concilio, Relat. Dioec. 479, "Relatio oratorum Moguntinorum", 10 octobre 1609, fo 68v : "Hic grassatione Brandeburgica iam pridem exustus ac aequatus [...] ex ipsis ruderibus excitari iterum ac magna impensa, memorabiliq[ue] opere, quod tamen ad exitum suum nondum peruenit, pro honore atq[ue] usu ipsius Ecclesiae reaedificari coeptus est".

27 ASV, Congregazione del concilio, Relat. Dioec. 479, "Moguntin[a] relatio quadrienny exhibita", 5 juillet 1615, fo 58 : "Nouam residentiam Archiepiscopalem in ciuitate Aschaffenburgi a Marchione Brandenburgico exustam [...] construxisse". 
monastères, des hospices et d'autres lieux pieux et religieux, menaçant ruine à cause de leur vétusté ou bien détruits et désolés en raison de l'injure du temps" 28 .

Cette ouvre de reconstruction ne se cantonne pas à la simple restauration mais touche également à la construction et à l'agrandissement d'autres bâtiments qui permettent de mieux saisir dans quelle mesure ces travaux édilitaires offrent à la fois la possibilité d'exercer un contrôle sur de possibles dissidences à venir et de jeter les bases d'une autorité dépassant les limites de la principauté mayençaise.

Ainsi, complétant les travaux concernant les résidences archiépiscopales, d'autres chantiers sont ouverts qui illustrent les nécessités d'un encadrement rénové de la population tant sur le plan spirituel que sur le temporel afin de fonder et renforcer la légitimité politique des princes, confrontée à des possibilités de contestation. L'introduction des ordres emblématiques de la Réforme catholique en offre un bon exemple : en 1561, Daniel Brendel von Homburg fait venir des jésuites à Mayence afin de diriger l'université. En 1564, ces derniers fondent dans la cité rhénane un collège et en 1575 , ils en ouvrent un nouveau à Heiligenstadt.

Si les relations détaillent l'avancée des travaux, notamment du collège de Mayence (en 1609, "on travaille encore maintenant à l'agrandir, l'orner et le munir de nombreuses constructions" ${ }^{29}$ ), cet encadrement des jésuites ne correspond pas seulement à une tentative d'unification de territoires et de populations autour de la confession romaine; c'est également un moyen pour les archevêques de Mayence d'accroître leur prestige : toujours en 1609, Johann Schweikard von Cronberg note avec un certain orgueil que "la jeunesse afflue en grand nombre aussi bien des Gaules et de Pologne que de Belgique, de la Germanie et des contrées voisines" vers ses collèges tenus par les jésuites ${ }^{30}$. À l'exception d'Erfurt qui reste gagnée au luthéranisme, ce travail de recatholicisation permet aux archevêques de Mayence de consolider leur autorité confessionnelle et de donner un semblant d'unité à des territoires épars et disparates. Le lien entre encadrement religieux et contrôle des dissidences devient évident dans la relation de 1659, même si sa nature change. Après une première étape correspondant à l'installation d'ordres liés à la Réforme catholique dans les espaces les plus peuplés, c'est-àdire dans les villes, l'effort contre-réformateur se porte désormais sur les paroisses

28 ASV, Congregazione del concilio, Relat. Dioec. 479, "Relatio oratorum Moguntinorum”, 10 octobre 1609, $\mathrm{f}^{\circ} 68 \mathrm{v}$ : "Scholas, ecclesias, monasteria, hospitalia atq[ue] alia pia et religiosa loca vel vetustate collapsa, vel alicqui temporum iniuria disiecta ac desolata [...] liberaliter restaurat".

29 ASV, Congregazione del concilio, Relat. Dioec. 479, "Relatio oratorum Moguntinorum, 10 octobre 1609, f 71 : "In quo dilatando atq[ue] exornando, pluribusq[ue] structuris aptando etiamnum laboratur in praesens".

30 ASV, Congregazione del concilio, Relat. Dioec. 479, fo 22, relation ad limina des orateurs de Mayence, 1609 : "Magno numero Iuuentus tam ex Gallis et Polonia, quam ex Belgio atq[ue] ipsa Germania vicinisq[ue] partibus confluit". 
rurales et celles se situant à proximité de territoires luthériens et calvinistes ${ }^{31}$. Ici, le souci de contrôle de zones fragiles ou douteuses du point de vue de l'orthodoxie est patent. Mais il souligne également par contraste que les villes sont à la fois les sources d'où naissent les dissidences possibles et qu'elles constituent le facteur de déstabilisation le plus grand du fait de l'importance des populations concernées. Par ailleurs, à une autre échelle, le redressement de l'université et l'établissement de Gymnasia permet sans doute à Mayence d'élargir son rayonnement, non seulement à l'intérieur du Saint-Empire mais également au-delà des limites du monde germanique. De ce point de vue, la lutte contre les dissidences permet de changer la nature du pouvoir princier et contribue à l'inscrire dans une échelle de pouvoirs plus vastes avec lesquels il doit désormais s'articuler.

3. Parmi les raisons les plus évidentes pour expliquer ce fait, il faut souligner que les archevêques de Mayence participent à d'autres réseaux de pouvoirs qui leur permettent de construire et de renforcer leur légitimité à l'intérieur du Saint-Empire. Hormis le pouvoir impérial, ces sources de légitimité correspondent pour l'essentiel au pape. C'est une façon pour eux de maintenir et de conforter une position politique et institutionnelle au sein de l'espace impérial qui n'a rien de définitivement acquise. De ce point de vue, au-delà des menaces immédiates que peuvent représenter les dissidences dans les territoires urbains mayençais, leur résolution est également une façon de renforcer la légitimité des archevêques de Mayence. En se pliant aux exigences des relations ad limina et en mettant en scène la promotion d'un catholicisme inspiré des canons du concile de Trente, les archevêques de Mayence affirment leur orthodoxie religieuse et donc leur légitimité pastorale. Mais une telle affirmation ne se cantonne pas au seul domaine religieux. Dans la mesure où nombre de charges, y compris temporelles, doivent être confirmées par la papauté, le lien avec cette dernière est primordial pour construire le pouvoir des archevêques.

Ainsi, en 1569, Daniel Brendel von Homburg sollicite le cardinal Giovanni Francesco Commendone, alors en mission dans le Saint-Empire, afin que le pape reconnaisse deux nouveaux vicaires pour la Thuringe et la région rhénane. Pour l'archevêque, privé de ses adjoints "une fois que Dieu les a appelés à une autre vie" : "ils ont toujours été nécessaires à nos provinces, surtout en cette période où la vigilance, l'assiduité et le travail sont plus grands qu'auparavant, d'autant que ces temps sont plus troublés ${ }^{32}$. antehac vigilantia, industria, atq[ue] labore opus est, quanto tempora ipsa magis turbulenta". 
Il n'est ici pas seulement question de faire confirmer un ordre social mais d'obtenir les moyens de pouvoir gouverner un territoire éclaté qui requiert attention et compétence : en ces années 1560, la Thuringe est touchée par la réforme luthérienne qui est sur le point de devenir la confession majoritaire dans cette région et la partie rhénane de la principauté de Mayence voisine avec des territoires ouvertement passés au calvinisme, comme le Palatinat ou la Hesse. Dans cette perspective, la relation avec le pape, par l'intermédiaire de Commendone et du cardinal d'Augsbourg Otto Truchse $\beta$ von Waldburg, également sollicité, vise à assurer l'intégrité territoriale d'un État qui se sent menacé et les fondements de la puissance temporelle d'un prince ecclésiastique. Par ailleurs, à partir des années 1580, les lettres écrites à Mayence sont directement adressées à Grégoire XIII et non plus au cardinal protecteur de la Curie, preuve sans doute que la stratégie de renforcer les liens avec la papauté a porté ses fruits. L'un des objectifs de cette stratégie, en plus de renforcer le pouvoir archiépiscopal, est de faire reconnaître les prétentions des princes ecclésiastiques de Mayence au titre de primat de Germanie. Comme cela a déjà été souligné par l'historiographie de langue allemande, il s'agit d'une question essentielle pour les archevêques de Mayence : son enjeu n'est pas seulement d'obtenir une prééminence morale sur leurs homologues allemands mais de faire en sorte que cette autorité spirituelle soit mise au service d'un projet politique pour assurer leur position de deuxième homme du Saint Empire ${ }^{33}$. Ainsi, quel que soit le cadre envisagé, la relation entre la papauté et les archevêques de Mayence est essentielle à ce dernier pour leur permettre de construire et de renforcer leur légitimité à l'intérieur du Saint Empire. Cela est d'autant plus important que la première moitié du XvII ${ }^{\mathrm{e}}$ siècle est une période où la cohésion interne de l'Empire est mise à l'épreuve, notamment par la question confessionnelle. Dans ce contexte, la relation avec Rome fondée sur la lutte contre les dissidences confessionnelles à l'intérieur de la principauté ecclésiastique de Mayence permet aux archevêques de remédier partiellement à ce dernier inconvénient tout en confirmant leur importance institutionnelle dans le Saint-Empire. Dans un tel cadre, les relations ad limina servent également à mettre en scène l'efficacité de la lutte contre les dissidences religieuses et la parfaite orthodoxie tridentine des archevêques de Mayence.

Toutefois, la lutte contre les dissidences religieuses ne permet pas seulement aux archevêques de Mayence de renforcer leur relation avec la papauté, elle leur ouvre également la possibilité d'entrer en contact avec la Monarchie Catholique. Alors que le pouvoir de l'empereur est grandement fragilisé et que cette dernière est à la recherche d'alliés dont il s'agit de s'assurer de la fiabilité, la monarchie hispanique fait de l'appartenance confessionnelle et de la mise en œuvre des décrets tridentins des critères importants dans la définition des alliances dans l'espace ger-

33 MaY, “Der Erzbischof von Mainz als Primas”, pp. 63-68. 
manique. En agissant en prélats soucieux de la réforme intérieure de leur Église, les archevêques de Mayence donnent alors des gages de leur ancrage dans le camp catholique et de leur possible compatibilité avec les objectifs de la Monarchie Catholique.

Le changement de perception des archevêques par les agents du Roi Catholique témoigne de cette compatibilité. En 1604, au moment de son élection donc, Johann Schweikard von Cronberg s'attire cette description de l'ambassadeur espagnol dans le Saint-Empire, Guillén de San Clemente :

"C'est un homme bon et un bon catholique et très dévoué au service de la sérénissime Maison d'Autriche mais de plus de soixante ans et de peu de bien et dont on ne peut attendre qu'il œuvre activement pour prendre les résolutions qui s'imposent. Il conviendra bien à l'empereur parce que je pense qu'il ne le pressera pas plus que ce qu'il ne souhaite pour choisir un Roi des Romains, se contentant de s'être élevé du rang de pauvre chevalier à la dignité électorale ${ }^{34}$.

Malgré une tonalité globalement positive, il s'agit d'un discours a minima sur un individu qui semble bien disposé mais dont les relations avec les Espagnols sont limitées : son orthodoxie confessionnelle est relevée, de même que son inclination en faveur des Habsbourg d'Autriche pour la succession impériale.

Ce discours évolue au fur et à mesure que l'archevêque de Mayence fait la preuve de l'intérêt de son attitude pour les Espagnols, c'est-à-dire en luttant contre les dissidences, et qu'il se montre désireux d'intégrer le réseau des clients hispaniques. Quand il est demandé au grand commandeur de León, Juan de Idiáquez, d'évaluer l'intérêt d'accorder une pension à Johann Schweikard von Cronberg en 1608 pour les services rendus à Philippe III, il qualifie l'Archevêque Électeur de Mayence de "Prince puissant, particulièrement parmi les catholiques de l'Empire et en très grand crédit et de très grande autorité dans celui-ci, qui montre beaucoup de zèle envers la religion catholique et les affaires de sa Majesté et de la sérénissime maison [d'Autriche]"35.

34 AGS, E, leg. 708, n.f., lettre de Guillén de San Clemente à Philippe III, 20 mars 1604 : “Es buen hombre y buen catt[ólic] y afiçionado al serv[ici]o de la ser[enísi]ma Casa de Austria pero de más de 60 ańos de hedad y de poco bueno y que no se puede esperar del que apriete las resoluciones que se pretenden. Sera bien apropósito para el Emp[erad]or por q[ue] tengo por cierto q[ue] no le hará mas instançia de la q[ue] el querrá a que haga Rey de Romanos contentándose de aver subido de un pobre cavallero a la dignidad electoral".

35 AGS, E, leg. 2323, doc. 47, avis de Juan de Idiáquez, sans date mais vraisemblablement du début de 1608 : "El Arçobispo Elector de Maguncia ques principe poderoso y pr[imer]am[en]te entre los catt[óli] cos del Imperio y en el de muy grande credito y autoridad y quien tiene mucho zelo a la religion catt[óli]ca y a las cossas de su M[ajesda]d y desta ser[enísi]ma cassa”. 
L'appartenance confessionnelle et les bonnes dispositions, désormais étendues aux Habsbourg d'Espagne, envers la maison d'Autriche, sont reprises mais les Espagnols sont maintenant en mesure d'évaluer l'importance de l'archevêque dans son contexte impérial. Il s'agit d'une des conséquences indirectes et inattendues de la lutte menée par les archevêques de Mayence contre les dissidences religieuses qui ont pu toucher les cités de leur principauté : en s'affirmant comme des ecclésiastiques contre-réformateurs, les archevêques de Mayence sont parvenus à s'insérer dans les réseaux politiques et confessionnels catholiques qui structurent un vaste pan des relations entre les principautés européennes du début du XvII ${ }^{\mathrm{e}}$ siècle.

\section{Conclusions}

Les différents espaces urbains de la principauté ecclésiastique de Mayence sont touchés par des dissidences à la fois nombreuses et multiformes : elles peuvent être de nature politique, religieuse ou sociale. Très souvent, elles s'inscrivent dans un temps long et s'expriment sous des formes variées, même une fois considérées comme résolues. De ce point de vue, il ne faut pas traiter les dissidences comme des manifestations épisodiques et momentanées mais bien comme des phénomènes de plus longue durée à saisir dans toute leur épaisseur sociale.

Les espaces urbains apparaissent comme les espaces par excellence où naissent ces dissidences. La raison tient précisément à ce que les villes sont à la fois les points de convergence des richesses et des idées nouvelles et les lieux d'où le pouvoir politique s'exerce sur un territoire. De cette tension entre volonté d'encadrement et ferment de nouveauté sociale et culturelle naît la possibilité des dissidences.

L'irruption de ces dissidences dans les territoires mayençais répond à ce schéma. Mais il suscite également une réaction de la part des princes-archevêques dans le sens d'un accroissement du contrôle. On retrouve ici l'une des caractéristiques de la première modernité, le processus social de disciplinarisation ${ }^{36}$. À cet égard, les relations ad limina envoyées par les archevêques de Mayence durant la première moitié du XVII ${ }^{\mathrm{e}}$ siècle ne constituent pas seulement des témoignages de la mise en œuvre des décrets du concile de Trente. Dans la mesure où elles mettent en scène les méthodes déplayées pour lutter contre les dissidences et, parfois, les résultats obtenus, elles documentent les efforts déployés pour contenir et occulter du tissu urbain les traces de toute forme de contestation du pouvoir politique et religieux des archevêques.

36 Dans une littérature abondante, voir par exemple le travail fondateur de G. Oestreich, "Strukturprobleme des europäischen Absolutismus", dans Vierteljahresschrift für Sozial- und Wirtschaftsgeschichte, 55 (1969), p. 329-347 et l'article en espagnol de F. PaLOMo, "Disciplina christiana. Apuntes historiográficos en torno a la disciplina y el disciplinamiento social como categorías de la historia religiosa de la alta edad moderna", dans Cuadernos de Historia Moderna, 18 (1997) p. 119-138. 
Les effets de ces dissidences et de la lutte qu'elles suscitent ne sauraient être cantonnés à la seule question de l'exercice du pouvoir d'un prince sur son État. En imposant une reconfiguration du pouvoir et en réinscrivant ce dernier dans une géographie des pouvoirs à une échelle plus globale, les dissidences amènent les archevêques de Mayence à nouer des liens et à renforcer ceux déjà existant avec des acteurs étrangers à l'espace impérial. En parvenant à se forger l'image de princes triomphant des dissidences confessionnelles internes, les archevêques de Mayence sont en mesure de devenir des acteurs significatifs non seulement à l'échelle du Saint-Empire mais aussi du monde catholique européen. 
